\title{
BMJ Neurology Open Hospital diagnosed pneumonia before age 20 years and multiple sclerosis risk
}

Kelsi A Smith (D) ,1,2 Ayako Hiyoshi, ${ }^{3,4}$ Sarah Burkill, ${ }^{5,6}$ Shahram Bahmanyar,
Johan Öckinger, ${ }^{1,8}$ Lars Alfredsson, ${ }^{2,9}$ Tomas Olsson, ${ }^{10}$ Scott Montgomery ${ }^{1,3}$

\author{
ABSTRACT \\ Introduction Respiratory inflammation has been \\ proposed as a risk factor for MS. This study aims to \\ determine if hospital-diagnosed pneumonia in adolescence \\ (before age 20 years) is associated with subsequent \\ multiple sclerosis (MS).
}

Methods This case-control study included incident MS cases after age 20 years identified using the Swedish national registers. Cases were matched with 10 general population controls by age, sex and region. Pneumonia diagnoses were identified between 0-5, 6-10, 11-15 and 16-20 years of age. Conditional logistic regression models adjusted for infectious mononucleosis (IM) and education calculated ORs with $95 \% \mathrm{Cls}$. Urinary tract infections (UTIs), a common complication of MS, before age 20 years were included as a control diagnosis for reverse causation. Results There were 6109 cases and 49479 controls included. Pneumonia diagnosed between age 11-15 years was associated with subsequent MS (adj OR $2.00,95 \% \mathrm{Cl} 1.22$ to 3.27 ). Although not statistically significant, sensitivity analyses showed similar magnitude associations of pneumonia between age 11-15 years and MS. No statistically significant associations with MS for pneumonia at other age groups were observed. Adjustment for IM had no notable effect on associations, but was statistically significantly associated with MS. UTIs were not associated with MS.

Conclusion Pneumonia at 11-15 years of age was associated with MS, suggesting a possible role for inflammation of the respiratory system in the aetiology of MS during a period of susceptibility in adolescence. Further research on respiratory infections prior to MS onset should be conducted to replicate this finding and determine explanatory causal mechanisms.

\section{INTRODUCTION}

Check for updates

(C) Author(s) (or their employer(s)) 2020. Re-use permitted under CC BY-NC. No commercial re-use. See rights and permissions. Published by BMJ.

For numbered affiliations see end of article.

Correspondence to

Ms Kelsi A Smith;

kelsi.smith@ki.se
The lungs have constant contact with the exterior environment and are one of the body's first lines of defence against pathogens and substances causing inflammation and irritation. Evidence supports hypotheses that respiratory exposures causing inflammation are risk factors for multiple sclerosis (MS): a chronic, inflammatory, immune-mediated disease. Notable risks for MS include cigarette smoke exposure; ${ }^{1}$ while exposure to other lung irritants such as organic solvents have been suggested as risk factors. ${ }^{2}$ Smoking causes lung inflammation ${ }^{3}$ and interacts with MS risk genes. ${ }^{4}$

Evidence that the respiratory system may be implicated in development of neuroinflammation originates from animal models of autoimmune encephalomyelitis. Autoreactive $T$ cells enter the lungs and bronchusassociated lymphoid tissues (BALT) and are reprogrammed to gain a migratory profile that appears a prerequisite for crossing the blood-brain barrier. Thus, non-specific lung irritation could trigger resident autoreactive $\mathrm{T}$ cells in the lung, resulting in inflammation in the central nervous system (CNS). ${ }^{5}$ Several infectious agents have been investigated in MS and patients with MS are more likely to carry DNA from the intracellular airway pathogen, Chlamydia pneumoniae, in cerebrospinal fluid. ${ }^{6}$

We chose to study pneumonia rather than other respiratory infections as it has greater persistent inflammatory consequences in the lung than other common respiratory infections in children, ${ }^{7}$ and in adults is associated with new-onset cardiovascular and chronic kidney disease following a pneumonia episode. ${ }^{89}$ Adolescence may form part of a key risk period of MS susceptibility, prior to initiation of inflammation in the CNS, when risk factors could be increasing MS risk later in life. ${ }^{10}$ Environmental exposures in adolescence including infectious mononucleosis (IM) ${ }^{11}$ and traumatic brain injury are linked with subsequent MS risk. ${ }^{12}$ Here, we consider exposures in age groups approximately following periods of structural changes in the brain potentially relevant for susceptibility to exposures influencing MS risk.

There can be an extended preclinical phase (prodrome) following initiation of CNS autoimmunity before frank symptomatic MS onset and clinical diagnosis, resulting in increased hospital visits, especially for infection-related medical concerns. ${ }^{13}$ MS results in a greater burden of infections, particularly respiratory infections such as pneumonia. ${ }^{14}$ Therefore, 
we focus on pneumonia before age 20 years to reduce the possibility that pneumonia is a consequence of prodromal MS and include urinary tract infections (UTI) as a control diagnosis as they are also recognised complications of MS.

To the best of our knowledge, no studies have investigated the association of pneumonia in childhood and adolescence requiring hospital-care and MS risk using prospectively recorded data. This study aims to test the hypothesis that pneumonia before age 20 years is associated with subsequent MS, and that there may be variation by age at exposure using Swedish register data. We also considered IM diagnoses as, in theory, pneumonia could be secondary to this well-established MS risk factor.

\section{METHODS}

\section{Study population and data sources}

This case-control study, using Swedish national registers, identified all diagnoses of MS $(n=29310)$ recorded in either the National Patient Register (PR) or the Multiple Sclerosis Register (MSR) between 1968 and 2012. The PR identifies hospital discharge diagnoses with approximately $80 \%$ accuracy (with variation for specific diagnoses) since its implementation in 1964 with national coverage achieved in 1987 and outpatient diagnoses included since $2001 .^{15}$ It has fewer diagnostic errors among younger patients and a high level of accuracy for MS diagnoses. ${ }^{15}$ The MSR began in 1996 and has coverage of approximately $80 \%$ of all patients with MS in Sweden providing a high level of diagnostic accuracy ${ }^{16}$ with at least $96 \%$ having confirmed MS diagnoses. ${ }^{17}$ MS cases were individually matched with 10 (occasionally fewer where appropriate controls could not be identified) randomly selected general-population controls ( $n=293094)$ without MS at the MS case's earliest date of MS diagnosis (index date) from either the MSR or the PR. Matching was by birth year, sex, region (county) of residence and vital status (controls were matched only if alive) at index date using the Total Population Register. Cases and controls were excluded if born before 1964, or migrated to or from Sweden between birth and the earliest MS diagnosis date as identified in the Total Population Register (figure 1). Controls were excluded if their matched case was excluded. A total of 6685 cases and 54692 controls were included.

Pneumonia, IM and UTI diagnoses for both cases and controls were identified using International Classification of Disease (ICD) codes using up to five digits in the PR. Data linkage was achieved using the unique personal identification number issued to all Swedish residents.

\section{Patient and public participation}

There was no patient or public involvement in this study.

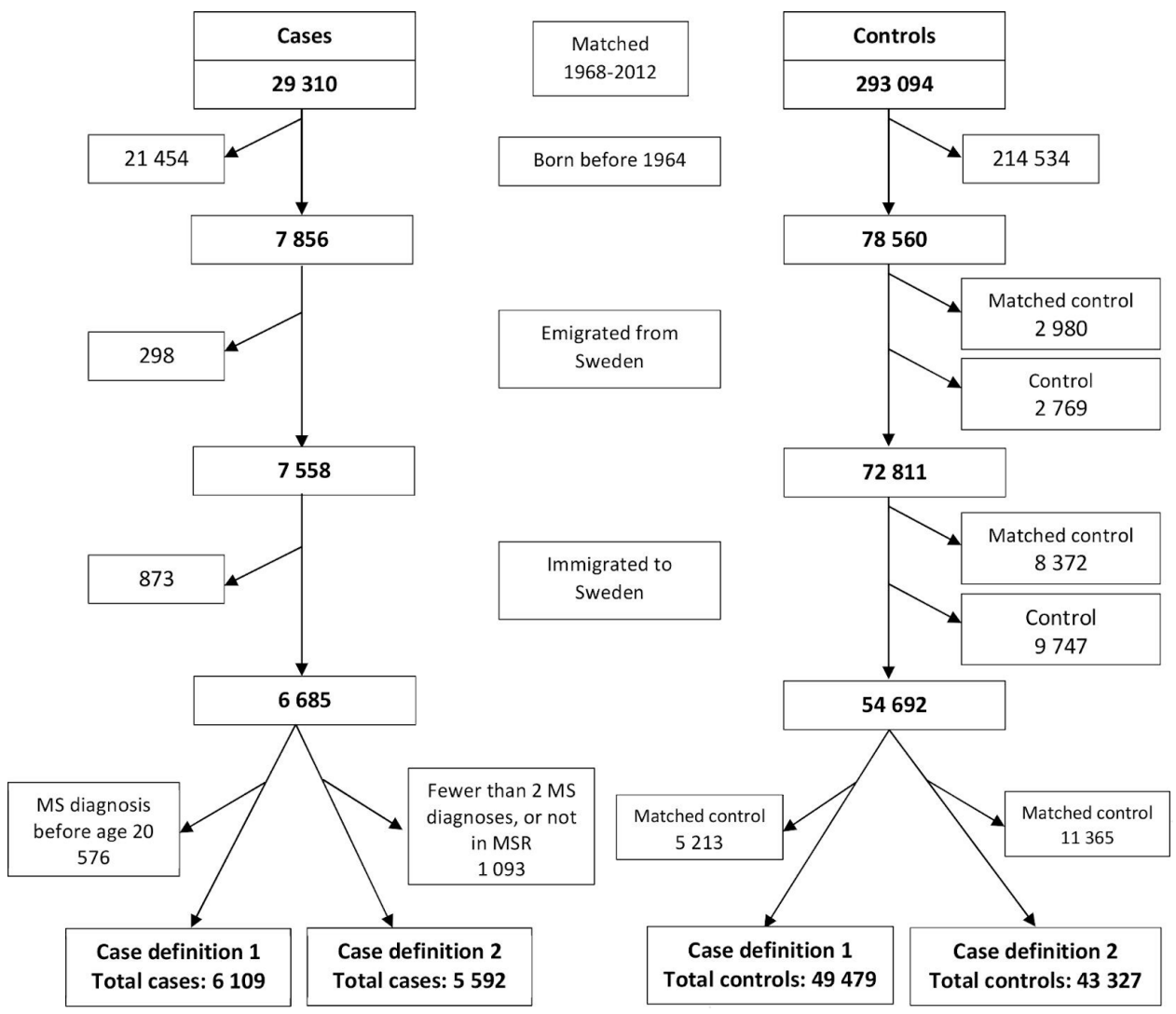

Figure 1 Definition of included cases using case definition 1 and included cases using case definition 2 identified from the Patient Register, and their matched controls from the Total Population Register. Case definition 1 is defined as at least one MS diagnosis over age 20 years from the National Patient Register (PR) or Multiple Sclerosis Register (MSR). Case definition 2 is defined as two or more MS diagnoses over age 20 years from the PR or diagnosis in the MSR. MS, multiple sclerosis. 


\section{Case and exposure definitions}

\section{Case definition: MS}

Incident MS diagnoses were identified in the PR using the earliest date of diagnosis using Swedish ICD codes (ICD-8 or ICD-9: 340, ICD-10: G35) between 1968 and 2012, or if included in the MSR. Two sets of inclusion criteria were used. Case definition 1 included all cases with a first MS diagnosis in the PR or inclusion in the MSR after 20 years of age (figure 1). Case definition 2 was additionally used to reduce potential misclassification of MS diagnoses, including cases with at least two MS diagnoses in the PR, or had a record in the MSR, with the first date of MS diagnosis after age 20 years.

\section{Exposure: Pneumonia and UTIS}

Pneumonia diagnoses between birth to 20 years of age were identified in the PR between 1964 and 2012 using Swedish ICD codes (ICD 7: 490-493, ICD-8: 480-486, ICD-9: 480-486, ICD-10: J12-J18) as either the primary or secondary discharge diagnosis. A control infectious disease of UTI was used to explore the possibility of reverse causation where prodromal MS or MS-related susceptibility increases the risk of infection before age 20 years. UTIs were chosen as they, like pneumonia, are typically more common among patients with MS and can be a complication of MS. ${ }^{18}$ A primary/secondary diagnosis of UTI was identified from the PR using Swedish ICD codes (ICD 7: 600, 605; ICD-8: 590, 595, 599.02; ICD-9: 590, 595, 599.0; ICD-10: N30, N39, N10). Separate episodes of pneumonia and UTIs were defined as a minimum of 90 days between diagnoses. Diagnoses were categorised into age groups of $0-5,6-10,11-15$ and $16-20$ years of age, and were dichotomised as ever/never diagnosed due to sparse data. These age groups were chosen given infants and children between $0-5$ years of age have higher risk of developing pneumonia, and second to approximately correspond to developmental phases of the CNS: 0-5 years (early rapid development); 6-10 years (beginnings of structural reorganisation approaching puberty); 11-15 years (peripubertal reorganisation and synaptogenesis) ${ }^{19}$ and 16-20 years (synaptic pruning and increased axonal myelination) $)^{20-22}$ that may be relevant for susceptibility influencing MS risk.

\section{Potential confounding factors}

Year of birth, sex and region of residence were matching factors because they are important potential confounding factors due to age and sex differences in MS and pneumonia, as well as regional variation in diagnosis rates and healthcare usage. Highest education level obtained from the Longitudinal Integrated Database for Health Insurance and Labour Market Studies (Swedish acronym, LISA) was also considered as educational level is an indicator of socioeconomic conditions and has been associated with $\mathrm{MS}^{23}$ and infections. ${ }^{24}$ Education level was classified into compulsory school education or less (mandatory school attendance to age 15 years); postcompulsory secondary school; postsecondary education such as college or university and educational data unavailable.

IM as a primary/secondary diagnosis was identified in the PR by ICD codes (ICD-7: 093; ICD-8: 075; ICD-9: 075; ICD-10: B27) between 1964 and 2012 and classified as ever/never diagnosed in four age categories: 0-5, 6-10, 11-15 and 16-20 years of age. IM is a risk factor for MS and could potentially signal increased susceptibility to other infections. $^{25}$

\section{Statistical analysis}

Conditional logistic regression conditioned on the risk set (defined by matching characteristics) and adjusted for confounders was used to examine the association between pneumonia MS using ORs. Associations between IM and pneumonia were also tested using conditional logistic regression. Statistical significance was defined as 95\% CIs not including $1.00(\mathrm{p}<0.05)$.

Three sensitivity analyses were performed. In sensitivity analysis 1, pneumonia diagnoses were restricted to only primary diagnoses. In sensitivity analysis 2 , an additional age category of 20-30 years was added for pneumonia exposure and included MS diagnoses of over 30 years of age; and finally, sensitivity analysis 3 considered the appearance of clinical symptoms (onset) before a diagnosis of MS: the MSR includes a question for age at which the first suspected symptom of MS occurred, as symptoms of MS often occur many years before the first MS diagnosis. Therefore, only cases with both an MS diagnosis and an age at suspected onset after 20 years of age (and their matched controls) were included. These sensitivity analyses were performed in order to further exclude the possibility of reverse causation.

The analysis was conducted using SAS V.9.4 (SAS Institute, 2016) and Stata V.15 software (StataCorp, 2017).

\section{RESULTS}

\section{Baseline characteristics}

Case definition 1 (one diagnosis of MS in the PR or MSR inclusion after age 20 years) included 6109 cases with 49479 matched controls (figure 1), of which $70.68 \%$ were female and $46.55 \%$ had an index date between 30 and 39 years of age (table 1). Controls had higher educational level than cases, but fewer had been diagnosed with IM across all age groups than cases (table 2). Case definition 2 (2+MS diagnoses in the PR, or inclusion in the MSR) included 5592 cases and 43327 controls with similar characteristics as case definition 1. For both cases and controls, pneumonia diagnosis (primary or secondary) among both cases and controls were infrequent as $<1 \%$ were diagnosed in all age groups (table 2). As expected, the highest proportion of pneumonia diagnoses for both case definitions was between age $0-5$ years. Diagnoses of IM were also infrequent, with the highest proportion between 16-20 years of age, and more frequent among cases than controls. No association between IM and pneumonia was found. 
Table 1 Participant characteristics at the index date of MS individuals and matched controls

\begin{tabular}{|c|c|c|c|c|}
\hline \multirow[b]{2}{*}{ Characteristic } & \multicolumn{2}{|c|}{ Case definition $1^{*}$} & \multicolumn{2}{|c|}{ Case definition $2 \dagger$} \\
\hline & Cases n (\%) & Controls n (\%) & Cases (\%) & Controls (\%) \\
\hline Total study population & 6109 & 49479 & 5592 & 43327 \\
\hline \multicolumn{5}{|l|}{ Sex $\ddagger$} \\
\hline Male & 1791 (29.32) & 14705 (29.72) & $1601(28.63)$ & $12550(28.97)$ \\
\hline Female & $4318(70.68)$ & 34774 (70.28) & 3991 (71.37) & $30777(71.03)$ \\
\hline \multicolumn{5}{|l|}{ Year of birth $\ddagger$} \\
\hline $1964-1968$ & $2032(33.26)$ & 16686 (33.72) & 1855 (33.17) & 14715 (33.96) \\
\hline 1969-1973 & $1575(25.78)$ & 12793 (25.86) & 1451 (25.95) & $11326(26.14)$ \\
\hline $1974-1978$ & $1226(20.07)$ & 9864 (19.94) & 1115 (19.94) & 8685 (20.05) \\
\hline $1979-1983$ & $825(13.50)$ & $6450(13.04)$ & $766(13.7)$ & $5602(12.93)$ \\
\hline $1984-1988$ & $370(6.06)$ & $3002(6.07)$ & 333 (5.95) & $2482(5.73)$ \\
\hline 1989-1991 & $81(1.33)$ & $684(1.38)$ & $72(1.29)$ & $517(1.19)$ \\
\hline \multicolumn{5}{|l|}{ Age (years) at index $\neq$} \\
\hline $20-29$ & 2327 (38.09) & 19458 (39.33) & 2149 (38.43) & 17308 (39.95) \\
\hline 30-39 & 2844 (46.55) & 22559 (45.59) & $2607(46.62)$ & $19947(46.04)$ \\
\hline $40-49$ & $938(15.35)$ & $7462(15.08)$ & 836 (14.95) & $6072(14.01)$ \\
\hline \multicolumn{5}{|l|}{ Education } \\
\hline Compulsory & $482(7.89)$ & 3459 (6.99) & $426(7.62)$ & 3039 (7.01) \\
\hline Postcompulsory & 2938 (48.09) & 23428 (47.35) & $2678(47.89)$ & $20410(47.11)$ \\
\hline Postsecondary & 2665 (43.62) & 22464 (45.4) & $2471(44.19)$ & 19768 (45.63) \\
\hline Unknown & $24(0.39)$ & $128(0.26)$ & $17(0.3)$ & $110(0.25)$ \\
\hline
\end{tabular}

${ }^{*}$ Case definition 1: at least one MS diagnosis over age 20 years from the PR or MSR.

†Case definition 2: two or more MS diagnoses over age 20 years from the PR or diagnosis in the MSR.

$\ddagger$ Matching variable.

MSR, Multiple Sclerosis Register; PR, National Patient Register.

\section{Pneumonia as a primary or secondary diagnosis between birth and age 20 years}

A primary/secondary diagnosis of pneumonia between 11-15 years of age was statistically significantly associated with MS after 20 years of age (OR 2.00, 95\% CI: 1.22 to 3.27) (table 2). Pneumonia diagnoses in all other age groups showed no statistically significant association with MS. Estimates remained similar after adjustment for IM and education. Consistent with previous research, IM in all age groups after age 6 years was statistically significantly associated with MS. The highest magnitude association of IM with MS was among individuals aged 11-15 years (adj OR 2.77, 95\% CI: 1.89 to 4.06). UTIs were infrequent, and showed no association with MS. Post hoc analyses showed from age 6 years onwards, very few males had UTI diagnoses and in females UTI diagnoses were apparent after age 11 years, although few.

Using case definition 2, the magnitude of the association of pneumonia diagnosed between 11-15 years in cases compared with controls was statistically significant, although somewhat reduced (adj OR 1.90, 95\% CI: 1.12 to 3.23) after full adjustment (table 2). Results for the age groups $0-5,6-10,16-20)$ were similar to those for case definition 1, showing no association of pneumonia with MS and statistically significant associations of IM with MS.

\section{Sensitivity analysis 1: pneumonia as a primary diagnosis between birth and age 20 years}

Restricting the exposure to pneumonia as a primary diagnosis showed similar association with MS in the 11-15 age group using case definition 1 with a primary/secondary pneumonia diagnosis. Pneumonia diagnosed between 11-15 years was associated with MS after adjustment for IM and education (adj OR 2.00, 95\% CI: 1.13 to 3.52) (table 3). IM diagnosed in adolescence showed an association with MS, especially for IM between 11-15 years (adj OR 2.76, 95\% CI: 1.89 to 4.05). No other statistically significant associations of pneumonia with MS were observed. Using case definition 2, associations of pneumonia diagnosed between 11-15 years with MS were reduced in magnitude and without statistical significance after full adjustment (adj OR 1.78, 95\% CI: 0.95 to 3.33). IM associations remained similar to main analyses.

\section{Sensitivity analysis 2: first MS diagnosis after age $\mathbf{3 0}$ years}

Restricting MS diagnosis to after age 30 years showed similar magnitude association of pneumonia between 
Table 2 The association of pneumonia as a primary or secondary diagnosis between birth and age 20 years and multiple sclerosis diagnosed over 20 years of age

\begin{tabular}{|c|c|c|c|c|c|c|}
\hline Age group & Cases n (\%) & Cont n (\%) & Model 1 OR $(95 \% \mathrm{Cl})^{*}$ & Model 2 OR $(95 \% \mathrm{Cl})^{*}$ & Model 3 OR $(95 \%$ Cl)* & Model 4 OR $(95 \% \mathrm{Cl})^{*}$ \\
\hline Case definition $1 \dagger$ & 6109 & 49479 & & & & \\
\hline \multicolumn{7}{|l|}{ Pneumonia } \\
\hline $6-10$ & $16(0.26)$ & $161(0.33)$ & 0.80 (0.48 to 1.34$)$ & 0.79 (0.47 to 1.33$)$ & 0.79 (0.47 to 1.33$)$ & 0.79 (0.47 to 1.33$)$ \\
\hline $11-15$ & $20(0.33)$ & $81(0.16)$ & 2.00 (1.22 to 3.27$)$ & 2.00 (1.22 to 3.27$)$ & 2.02 (1.23 to 3.30$)$ & 2.00 (1.22 to 3.27$)$ \\
\hline $0-5$ & $6(0.1)$ & $21(0.04)$ & & 2.14 (0.86 to 5.33$)$ & 2.13 (0.86 to 5.31$)$ & 2.14 (0.86 to 5.34$)$ \\
\hline $6-10$ & $9(0.15)$ & $37(0.07)$ & & 1.90 (0.91 to 3.94$)$ & 1.89 (0.91 to 3.94$)$ & 1.93 (0.93 to 4.00$)$ \\
\hline $11-15$ & $36(0.59)$ & $106(0.21)$ & & 2.76 (1.89 to 4.04$)$ & 2.77 (1.89 to 4.05$)$ & 2.77 (1.89 to 4.06$)$ \\
\hline $16-20$ & $51(0.83)$ & $178(0.36)$ & & 2.28 (1.66 to 3.12$)$ & 2.27 (1.66 to 3.11$)$ & 2.29 (1.68 to 3.14$)$ \\
\hline $16-20$ & $60(0.98)$ & $362(0.73)$ & & & $1.32(1.00$ to 1.74$)$ & $1.30(0.98$ to 1.71$)$ \\
\hline Case definition $2 \ddagger$ & 5592 & 43327 & & & & \\
\hline \multicolumn{7}{|l|}{ Pneumonia } \\
\hline $0-5$ & $46(0.82)$ & $428(0.99)$ & 0.84 (0.61 to 1.15$)$ & 0.83 (0.61 to 1.14$)$ & $0.83(0.61$ to 1.14$)$ & $0.83(0.6$ to 1.13$)$ \\
\hline $6-10$ & $15(0.27)$ & $147(0.34)$ & 0.77 (0.44 to 1.33$)$ & $0.77(0.44$ to 1.34$)$ & 0.77 (0.44 to 1.34$)$ & 0.77 (0.44 to 1.34$)$ \\
\hline $11-15$ & $17(0.3)$ & $74(0.17)$ & 1.88 (1.11 to 3.19$)$ & 1.87 (1.10 to 3.18$)$ & 1.91 (1.13 to 3.25$)$ & 1.90 (1.12 to 3.23$)$ \\
\hline $16-20$ & $17(0.3)$ & $94(0.22)$ & 1.32 (0.77 to 2.29$)$ & 1.28 (0.74 to 2.22$)$ & 1.28 (0.74 to 2.22$)$ & 1.27 (0.73 to 2.19$)$ \\
\hline \multicolumn{7}{|l|}{ IM } \\
\hline $0-5$ & $5(0.09)$ & $20(0.05)$ & & 1.57 (0.54 to 4.60$)$ & 1.56 (0.53 to 4.57$)$ & 1.58 (0.54 to 4.63$)$ \\
\hline $11-15$ & $7(0.13)$ & $94(0.22)$ & & & 0.53 (0.25 to 1.16$)$ & $0.53(0.24$ to 1.14$)$ \\
\hline $16-20$ & $54(0.97)$ & $317(0.73)$ & & & 1.32 (0.98 to 1.78$)$ & $1.30(0.96$ to 1.75$)$ \\
\hline
\end{tabular}

Bold text indicates results where the $95 \% \mathrm{Cl}$ does not cross 1.00 .

*Model 1: adjusted for matching variables; model 2: adjusted for matching variables and IM; model 3: adjusted for matching variables, IM and UTIs; model 4: adjusted for matching variables, IM and education. In all models, the OR is comparing having a diagnosis within the age group with not having a diagnosis in the age group.

†Case definition 1: at least one MS diagnosis over age 20 years from the PR or MSR.

fCase definition 2: two or more MS diagnoses over age 20 years from the PR or diagnosis in the MSR.

Cont, controls; IM, infectious mononucleosis; MSR, Multiple Sclerosis Register; $n$, total individuals in group; PR, National Patient Register; UTI, urinary tract infection.

ages 11-15 years using case definition 1 and 2 with MS (table 4), although the association was not statistically significant and was marginally attenuated compared with the main analysis (table 2). No other statistically significant associations of pneumonia with MS were found. Pneumonia diagnoses between 21-30 years showed no association with MS. IM from age 11 years onwards was statistically significantly associated with MS. It should be noted due to restriction of MS diagnosis after age 30 years, data became sparse and CIs wider.

\section{Sensitivity analysis 3 : MS onset and diagnosis after age 20 years}

Restricting MS cases (and matched controls) to individuals diagnosed and with onset after age 20 years showed similar associations of pneumonia and IM with MS as previously observed, but statistical power was reduced resulting in lower magnitude point estimates and nonstatistically significant pneumonia results (table 5). Primary/secondary pneumonia diagnosis between 11-15 years of age showed non-statistically significant 
Table 3 Sensitivity analysis 1: the association of pneumonia as a primary diagnosis between birth and age 20 years and multiple sclerosis diagnosed over 20 years of age

\begin{tabular}{|c|c|c|c|c|c|}
\hline Age group & Cases n (\%) & Cont n (\%) & Model 1 OR $(95 \% \mathrm{Cl})^{*}$ & Model 2 OR $(95 \% \mathrm{Cl})^{*}$ & Model 3 OR $(95 \% \mathrm{Cl})^{*}$ \\
\hline Case definition 1† & 6109 & 49479 & & & \\
\hline \multicolumn{6}{|c|}{ Pneumonia } \\
\hline $0-5$ & $42(0.69)$ & $378(0.76)$ & 0.89 (0.65 to 1.23$)$ & 0.89 (0.64 to 1.23$)$ & 0.88 (0.64 to 1.22$)$ \\
\hline $6-10$ & $14(0.23)$ & $122(0.25)$ & 0.92 (0.53 to 1.60$)$ & 0.91 (0.52 to 1.58$)$ & 0.91 (0.52 to 1.58$)$ \\
\hline $11-15$ & $15(0.25)$ & $60(0.12)$ & 2.00 (1.13 to 3.53$)$ & 2.01 (1.14 to 3.54$)$ & 2.00 (1.13 to 3.52$)$ \\
\hline $16-20$ & $15(0.25)$ & $99(0.2)$ & 1.25 (0.72 to 2.15$)$ & 1.20 (0.70 to 2.08$)$ & 1.18 (0.69 to 2.05$)$ \\
\hline \multicolumn{6}{|l|}{ IM } \\
\hline $0-5$ & $6(0.1)$ & $21(0.04)$ & & 2.14 (0.86 to 5.33$)$ & 2.15 (0.86 to 5.36$)$ \\
\hline $6-10$ & $9(0.15)$ & $37(0.07)$ & & 1.90 (0.91 to 3.94$)$ & 1.93 (0.93 to 4.01$)$ \\
\hline $11-15$ & $36(0.59)$ & $106(0.21)$ & & 2.76 (1.89 to 4.04$)$ & 2.76 (1.89 to 4.05$)$ \\
\hline $16-20$ & $51(0.83)$ & $178(0.36)$ & & 2.28 (1.66 to 3.12 ) & 2.30 (1.68 to 3.15$)$ \\
\hline Case definition $2 \ddagger$ & 5592 & 43327 & & & \\
\hline \multicolumn{6}{|c|}{ Pneumonia } \\
\hline $0-5$ & $37(0.66)$ & $323(0.75)$ & 0.88 (0.62 to 1.26$)$ & 0.87 (0.62 to 1.24$)$ & 0.87 (0.61 to 1.23$)$ \\
\hline $6-10$ & $13(0.23)$ & $111(0.26)$ & 0.87 (0.48 to 1.58$)$ & 0.88 (0.48 to 1.59$)$ & 0.88 (0.48 to 1.60$)$ \\
\hline $11-15$ & $12(0.21)$ & $54(0.12)$ & 1.78 (0.95 to 3.33$)$ & 1.79 (0.96 to 3.35$)$ & 1.78 (0.95 to 3.33$)$ \\
\hline $16-20$ & $13(0.23)$ & $85(0.2)$ & 1.08 (0.58 to 2.03 ) & 1.04 (0.55 to 1.96$)$ & 1.02 (0.54 to 1.93 ) \\
\hline \multicolumn{6}{|l|}{ IM } \\
\hline $0-5$ & $5(0.09)$ & $20(0.05)$ & & 1.57 (0.54 to 4.60$)$ & 1.59 (0.54 to 4.67$)$ \\
\hline $6-10$ & $9(0.16)$ & $27(0.06)$ & & 2.35 (1.06 to 5.18 ) & 2.38 (1.08 to 5.26$)$ \\
\hline $11-15$ & $36(0.64)$ & $93(0.21)$ & & 2.98 (2.01 to 4.44$)$ & 2.99 (2.01 to 4.44$)$ \\
\hline $16-20$ & $47(0.84)$ & $150(0.35)$ & & 2.33 (1.66 to 3.27$)$ & 2.35 (1.67 to 3.29$)$ \\
\hline
\end{tabular}

Bold text indicates results where the $95 \% \mathrm{Cl}$ does not cross 1.00 .

*Model 1: adjusted for matching variables; model 2: adjusted for matching variables and IM; model 3: adjusted for matching variables, IM and education. In all models, the OR is comparing having a diagnosis within the age group with not having a diagnosis in the age group.

†Case definition 1: at least one MS diagnosis over age 20 years from the PR or MSR.

$\ddagger$ Case definition 2: two or more MS diagnoses over age 20 years from the PR or diagnosis in the MSR.

Cont, controls; IM, infectious mononucleosis; MSR, Multiple Sclerosis Register; $n$, total individuals in group; PR, National

Patient Register.

associations with MS (OR 1.72, 95\% CI: 0.95 to 3.14 ) and after adjustment for IM and education (adj OR 1.71, 95\% CI: 0.94 to 3.12$)$.

\section{DISCUSSION}

Diagnosis of pneumonia between 11-15 years of age was associated with MS after age 20 years. The magnitude of the association remained similar, although somewhat reduced and not statistically significant, after imposing stricter inclusion criteria and multiple sensitivity analyses including accounting for possible MS onset.

From exposure to risk factors, to initiation of MS disease activity, it may take 10-20 years before there is sufficient clinical information to make an MS diagnosis. ${ }^{26}$ Adolescence likely represents a critical period of susceptibility before MS's onset or prodromal phase when some environmental exposures may initiate autoimmune processes in the CNS resulting in MS development. ${ }^{12} 27$
We hypothesise that pneumonia infections in adolescence could represent such an exposure.

\section{Association of pneumonia with subsequent MS}

Only pneumonia between ages 11-15 years was statistically significantly associated with subsequent MS. This is consistent with other studies showing some MS risks in adolescence are not associated with MS if they occur in earlier childhood, such as Epstein-Barr virus infections/ IM, ${ }^{11} 28$ or CNS trauma. ${ }^{12} 29$ Additionally, IM is a known risk factor for MS and the largest magnitude association between IM and MS seen in this study was for IM infection at $11-15$ years. This possibly indicates heightened MS susceptibility during the peripubertal period, which may help to explain the association with pneumonia at this age with subsequent MS. The sensitivity analysis with MS diagnosis after age 30 years found similar results (although of smaller magnitude and not statistically significant) for peripubertal pneumonia, while pneumonia at age 21-30 
Table 4 Sensitivity analysis 2: the association of a primary or secondary diagnosis of pneumonia between birth and age 30 years and multiple sclerosis diagnosed over 30 years of age

\begin{tabular}{|c|c|c|c|c|c|}
\hline Age group & Cases n (\%) & Cont n (\%) & Model 1 OR $(95 \% \mathrm{Cl})^{*}$ & Model 2 OR $(95 \% \mathrm{Cl})^{*}$ & Model 3 OR $(95 \%$ Cl)* \\
\hline Case definition $1 \dagger$ & 3457 & 27462 & & & \\
\hline \multicolumn{6}{|l|}{ Pneumonia } \\
\hline $0-5$ & $21(0.61)$ & $157(0.57)$ & 1.03 (0.65 to 1.62$)$ & 1.03 (0.65 to 1.63$)$ & 1.01 (0.64 to 1.60$)$ \\
\hline $6-10$ & $10(0.29)$ & $82(0.3)$ & $0.92(0.47$ to 1.77$)$ & 0.92 (0.47 to 1.78$)$ & 0.92 (0.48 to 1.79$)$ \\
\hline $11-15$ & $11(0.32)$ & $48(0.17)$ & 1.77 (0.92 to 3.42 ) & 1.79 (0.92 to 3.45$)$ & 1.75 (0.90 to 3.38 ) \\
\hline $16-20$ & $11(0.32)$ & $58(0.21)$ & 1.50 (0.78 to 2.87$)$ & 1.44 (0.75 to 2.77$)$ & 1.43 (0.74 to 2.74$)$ \\
\hline $21-30$ & $20(0.58)$ & $162(0.59)$ & 0.98 (0.62 to 1.56$)$ & 0.99 (0.62 to 1.57$)$ & 0.95 (0.60 to 1.52$)$ \\
\hline \multicolumn{6}{|l|}{ IM } \\
\hline $0-5$ & $1(0.03)$ & $11(0.04)$ & & 0.65 (0.08 to 5.04$)$ & 0.65 (0.08 to 5.05$)$ \\
\hline $6-10$ & $5(0.14)$ & $15(0.05)$ & & 2.31 (0.83 to 6.42 ) & 2.36 (0.85 to 6.57$)$ \\
\hline $11-15$ & $20(0.58)$ & $64(0.23)$ & & 2.50 (1.51 to 4.14$)$ & 2.50 (1.51 to 4.15$)$ \\
\hline $16-20$ & $32(0.93)$ & $74(0.27)$ & & 3.29 (2.17 to 4.99$)$ & 3.34 (2.20 to 5.08$)$ \\
\hline $21-30$ & $13(0.38)$ & $40(0.15)$ & & 2.60 (1.39 to 4.88$)$ & 2.61 (1.39 to 4.89 ) \\
\hline Case definition $2 \ddagger$ & 3145 & 23736 & & & \\
\hline \multicolumn{6}{|c|}{ Pneumonia } \\
\hline $0-5$ & $20(0.64)$ & $127(0.05)$ & 1.17 (0.72 to 1.90$)$ & $1.17(0.72$ to 1.91$)$ & 1.16 (0.71 to 1.89$)$ \\
\hline $6-10$ & $9(0.29)$ & $73(0.03)$ & 0.90 (0.45 to 1.82$)$ & $0.92(0.46$ to 1.85$)$ & 0.93 (0.46 to 1.86$)$ \\
\hline $11-15$ & $9(0.29)$ & $45(0.02)$ & 1.56 (0.76 to 3.21$)$ & $1.58(0.77$ to 3.23$)$ & 1.54 (0.75 to 3.17 ) \\
\hline $16-20$ & $11(0.35)$ & $48(0.02)$ & 1.53 (0.74 to 3.12$)$ & 1.44 (0.70 to 2.97$)$ & 1.42 (0.69 to 2.93 ) \\
\hline $21-30$ & $18(0.57)$ & $139(0.06)$ & 1.04 (0.64 to 1.71$)$ & 1.05 (0.64 to 1.72$)$ & 1.02 (0.62 to 1.68$)$ \\
\hline \multicolumn{6}{|l|}{ IM } \\
\hline $0-5$ & $1(0.03)$ & $11(0.00)$ & & / & / \\
\hline $6-10$ & $5(0.16)$ & $10(0.00)$ & & 3.75 (1.28 to 11.03$)$ & 3.83 (1.30 to 11.26$)$ \\
\hline $11-15$ & $20(0.64)$ & $55(0.02)$ & & 2.59 (1.51 to 4.43 ) & 2.60 (1.52 to 4.45$)$ \\
\hline $16-20$ & $30(0.95)$ & $62(0.03)$ & & 3.55 (2.28 to 5.53 ) & 3.60 (2.31 to 5.62 ) \\
\hline $21-30$ & $11(0.35)$ & $32(0.01)$ & & 2.80 (1.41 to 5.56$)$ & 2.78 (1.40 to 5.54$)$ \\
\hline
\end{tabular}

Bold text indicates results where the $95 \% \mathrm{Cl}$ does not cross 1.00 .

*Model 1: adjusted for matching variables; model 2: adjusted for matching variables and IM; model 3: adjusted for matching variables, IM and education. In all models, the OR is comparing having a diagnosis within the age group with not having a diagnosis in the age group.

†Case definition 1: at least one MS diagnosis over age 30 years from the PR or MSR.

¥Case definition 2: two or more MS diagnoses over age 30 years from the PR or diagnosis in the MSR.

Cont, controls; IM, infectious mononucleosis; MSR, Multiple Sclerosis Register; $n$, total individuals in group; PR, National Patient Register.

years was not associated with MS. We would expect an association of pneumonia during 21-30 years of age with MS after age 30 years due to possible influences of prodromal MS disease, which we did not observe, further indicating that the peripubertal period could be a critical period of susceptibility for MS development later in life. When including MS diagnosis and onset after age 20 years, the magnitude of association with MS between 11-15 years of age remained very similar, although not statistically significant, probably due to the notable decrease in statistical power (only a limited subset of patients with MS had information on age of onset). UTI diagnoses were even rarer than pneumonia during childhood and adolescence, but there was no evidence of an association with MS for this control diagnosis, a known consequence of MS. This further indicates that the association of pneumonia with MS is possibly not due to reverse causation during a period of prodromal MS disease activity.

\section{Possible role of pneumonia in development of MS}

The role of pneumonia in the development of MS is speculative, but pneumonia causes inflammation in the lung, and is associated with an influx of immune cells including $\mathrm{T}$ and $\mathrm{B}$ cells. We hypothesise that immune cells activated in response to lower airway pathogens are also involved in immune mechanisms related to MS. For example, airway infections by Streptococcus pneumoniae $e^{30} 31$ and Haemophilus influenzae ${ }^{32}$ induce a robust local and systemic T-helper (Th)17 response, including memory Th17 cells. Through molecular mimicry or other mechanisms, Th17 could be reactivated and infiltrate the CNS. ${ }^{33}$ Similarly, pneumonia is also associated with secretion of 
Table 5 Sensitivity analysis 3: the association of pneumonia between birth to age 20 years and multiple sclerosis with onset and diagnosis over 20 years of age

\begin{tabular}{|c|c|c|c|c|c|}
\hline \multicolumn{6}{|c|}{ Primary or secondary diagnosis of pneumonia } \\
\hline \multirow[t]{2}{*}{ Age group } & Cases n (\%) & Cont n (\%) & Model 1 OR $(95 \% \mathrm{Cl})^{*}$ & Model 2 OR $(95 \% \mathrm{Cl})^{*}$ & Model 3 OR $(95 \% \mathrm{Cl})^{\star}$ \\
\hline & 4244 & 34250 & & & \\
\hline $0-5$ & $36(0.85)$ & $337(0.98)$ & 0.86 (0.61 to 1.22$)$ & $0.86(0.60$ to 1.21$)$ & $0.86(0.60$ to 1.21$)$ \\
\hline $6-10$ & $9(1.07)$ & $108(0.32)$ & $0.66(0.34$ to 1.31$)$ & 0.67 (0.34 to 1.33$)$ & 0.67 (0.34 to 1.34$)$ \\
\hline $16-20$ & $13(3.07)$ & $75(0.22)$ & 1.43 (0.79 to 2.57$)$ & 1.39 (0.77 to 2.51$)$ & $1.38(0.76$ to 2.5$)$ \\
\hline \multicolumn{6}{|l|}{ IM } \\
\hline $0-5$ & $3(0.07)$ & $17(0.05)$ & & 1.34 (0.39 to 4.60$)$ & 1.34 (0.39 to 4.59$)$ \\
\hline $6-10$ & $6(0.14)$ & $25(0.07)$ & & 1.85 (0.75 to 4.53$)$ & $1.86(0.76$ to 4.55$)$ \\
\hline $11-15$ & $31(0.73)$ & $72(0.21)$ & & 3.44 (2.25 to 5.25$)$ & 3.44 (2.25 to 5.25$)$ \\
\hline \multicolumn{6}{|l|}{ Pneumonia } \\
\hline $0-5$ & $31(0.73)$ & $253(0.74)$ & 0.99 (0.68 to 1.44$)$ & 0.98 (0.67 to 1.42$)$ & 0.98 (0.67 to 1.42$)$ \\
\hline $6-10$ & $9(0.21)$ & $82(0.24)$ & 0.87 (0.43 to 1.73$)$ & 0.87 (0.43 to 1.74$)$ & 0.88 (0.44 to 1.75$)$ \\
\hline $11-15$ & $11(0.26)$ & $49(0.14)$ & 1.77 (0.92 to 3.41$)$ & 1.79 (0.93 to 3.44$)$ & 1.78 (0.92 to 3.43$)$ \\
\hline $16-20$ & $10(0.24)$ & $69(0.2)$ & 1.20 (0.62 to 2.33$)$ & 1.16 (0.60 to 2.26$)$ & 1.16 (0.59 to 2.25$)$ \\
\hline \multicolumn{6}{|l|}{ IM } \\
\hline $0-5$ & $3(0.07)$ & $17(0.05)$ & & 1.33 (0.39 to 4.58$)$ & 1.33 (0.39 to 4.57$)$ \\
\hline $6-10$ & $6(0.14)$ & $25(0.07)$ & & 1.86 (0.76 to 4.57$)$ & 1.87 (0.76 to 4.59$)$ \\
\hline
\end{tabular}

Bold text indicates results where the $95 \% \mathrm{Cl}$ does not cross 1.00 .

*Model 1: adjusted for matching variables; model 2: adjusted for matching variables and IM; model 3: adjusted for matching variables, $\mathrm{IM}$ and education. In all models, the OR is comparing having a diagnosis within the age group with not having a diagnosis in the age group.

Cont, controls; IM, infectious mononucleosis; $n$, total individuals in group.

CXCL12 (stromal-derived factor 1 ),$^{34} 35$ and expression of the CXCL12 receptor CXCR4 which has recently been described as a signature feature of $\mathrm{T}$ cells involved in MS pathology. ${ }^{36}$ This indicates potential immunological mechanisms, and activation of cellular subsets, through which pneumonia could increase the risk of MS. Similar mechanisms could also be involved in the aggravation of symptoms, relapses and increase of disability that are seen in patients with MS with airway infections. ${ }^{37}$ The lung and BALT have been shown to be involved in the initiation of autoimmune neuro-inflammation, ${ }^{5}$ however the role of BALT in humans is unclear as there is evidence indicating that BALT represents a constitutive structure in children, but in adults it is usually only present during pneumonia and in smokers. ${ }^{38-40}$

\section{Strengths and limitations}

This study determined age-specific exposures relevant to MS risk at ages that broadly represent stages of neurological development, assessing potentially critical periods before the preclinical phase of MS. The use of the PR captured serious episodes of hospital-diagnosed pneumonia that may be more aggressive, as less severe pneumonia is treated at the primary care level and is not recorded in the PR. We chose pneumonia as this respiratory infection is more serious than, for example, influenza or bronchitis, with more possible sequelae including long-term inflammatory and systemic immunological consequences. However, it is possible that other respiratory tract infections also influence MS risk.

This study controlled for IM diagnoses, another infection in adolescence associated with increased MS risk later in life, but adjusting for IM had little impact on the magnitude of the association between pneumonia and subsequent MS. This study considered MS diagnoses after age 20 years, and employed two strict inclusion criteria of MS cases in order to reduce misclassification and identify possible risk periods. It also included a sensitivity analyses using MS onset in conjunction with diagnosis and all analyses demonstrated a similar, consistent pattern of results across age groups. However, despite a reduction 
in numbers of subjects and episodes of pneumonia due to the stricter inclusion definitions, the magnitude of the association remained consistent, although somewhat reduced.

Potential limitations include possible misclassification of MS cases. Under case definition 1, use of both the PR and MSR captured more MS cases. Although the MSR has a high coverage, some patients are only captured by the PR. The PR has high accuracy of MS diagnoses, even when using one diagnosis of MS, but it is possible to receive an ICD code for suspected rather than confirmed MS, which may increase misclassification. This is why we also used case definition 2 as it has been shown that using a definition of two or more MS diagnoses in the PR reduces misclassification. ${ }^{41}$ Misclassification is mainly an issue from 2001 onwards due to the addition of the outpatient register to the PR. In a post hoc analysis of the MS cases with a first diagnosis from 2001 onwards under case definition $1(\mathrm{n}=4986), 90.77 \%$ were still included under case definition $2(\mathrm{n}=4526)$, demonstrating a high level of accuracy in this time period. Overall, $91.54 \%$ of all cases included under case definition 1 were also included under case definition 2, indicating a low risk of substantial misclassification bias. Additionally, the measure of MS onset may not always capture true onset of the disease process, as it is based on a history which may not identify symptoms truly associated with MS or the first symptom, but this is unlikely to overestimate risk. This measure was also only available for a subset of patients, notably reducing statistical power.

Furthermore, we cannot rule out that the observed association was potentially due to chance or unobserved confounding as we did not have information on lifestyle factors as our study was limited to register-based data. Despite factors such as active/passive smoking and obesity being unavailable, which could be associated with both pneumonia and MS, the association with pneumonia was largely limited to one age category, at an age before most people tend to start smoking heavily. Therefore, active/ passive smoking is unlikely to be a major confounding factor as the consequences of confounding of this nature would have been observed in most age groups (with associations of pneumonia with MS in most age groups), and especially in the age group 20-30 years as we would expect to see a possible cumulative effect of smoking over time. Nevertheless, we should consider these explanations given the primary analysis and sensitivity analysis 1 found an association of pneumonia between 11-15 years of age with MS, but observed non-statistically significant results in subsequent sensitivity analyses, although they were of a similar magnitude. This could also be due to reduced sample sizes after imposing stricter inclusion criteria and sensitivity analyses, losing nearly $50 \%$ of MS cases in sensitivity analysis 3 .

Another potential limitation included not being able to consider vaccinations against $S$. pneumoniae. Pneumococcal vaccination became part of the vaccination scheme in 2009, and little if any data are available before 2008 on the level of vaccination coverage in Sweden. ${ }^{42}$ However, this is unlikely to affect the results as too few of the individuals in this study would have been born sufficiently recently to have been vaccinated as children. Additionally, only up to a third of pneumonia diagnoses in unvaccinated children are caused by $S$. pneumoniae ${ }^{43}$ Furthermore, we could not assess if the cause of pneumonia (bacterial or viral) differentially influenced MS risk. Reliable differentiation of pneumonia diagnoses from the PR is difficult as not all specific infecting organisms are characterised, ICD codes vary over time and there is clinician and regional variation in coding.

Another potential limitation is the use of education as a proxy for socioeconomic circumstances: a more direct measure of material conditions of the household would be desirable to more accurately assess risk of infections.

\section{CONCLUSION}

This study strengthens the increasing evidence of a critical period in adolescence for later development of MS and also that the lungs, and serious respiratory infections or other sources of pulmonary inflammation, may play a role in MS aetiology. Further research examining associations of pneumonia and other severe respiratory infections with MS is needed to replicate these findings. If replicated, the role of these infections in MS aetiology should be determined.

\section{Author affiliations}

${ }^{1}$ Department of Medicine Solna, Karolinska Institutet, Stockholm, Sweden ${ }^{2}$ Institute of Environmental Medicine, Karolinska Institutet, Stockholm, Sweden ${ }^{3}$ Clinical Epidemiology and Biostatistics, Örebro Universitet-Campus USÖ, Orebro, Sweden

${ }^{4}$ Department of Public Health Sciences, Stockholm University, Stockholm, Sweden ${ }^{5}$ Centre for Pharmacoepidemiology, Karolinska Institutet, Stockholm, Sweden

${ }^{6}$ Saw Swee Hock School of Public Health, National University of Singapore, Singapore

${ }^{7}$ Centre for Psychiatry Research, Karolinska Institutet, Stockholm, Sweden ${ }^{8}$ Centre for Molecular Medicine, Karolinska Institutet, Stockholm, Sweden ${ }^{9}$ Centre for Occupational and Environmental Medicine, Stockholm, Sweden

${ }^{10}$ Department of Clinical Neuroscience, Karolinska Institutet, Stockholm, Sweden

Contributors KAS designed the study, analysed the data, produced and interpreted the results and wrote the manuscript. AH designed the study, performed preliminary data analysis and interpreted results and contributed to the manuscript. SBur managed and organised the register data, identified the study population and contributed to the methods and the manuscript. SBah was involved in conceptualising the hypothesis and research questions, designed the study and contributed to the manuscript. JÖ suggested potential immunological mechanisms and hypotheses, and contributed to the manuscript. LA and TO were involved in conceptualising the hypothesis and research questions, designed the study and contributed to the manuscript. SM conceptualised the hypothesis and research questions, designed the study, conducted preliminary data analysis, interpreted results and contributed to the manuscript.

Funding This study was funded by F. Hoffmann-La Roche (ZZK2PERAPP), Novartis Pharma (ZZK2PERAPP), Nyckelfonden (266331).

Competing interests TO has received MS research grants from the Swedish Research Council, the Swedish Brain Foundation and Margaretha af Ugglas Foundation. SM has received research funding from F. Hoffmann-La Roche, Novartis Pharma AG and AstraZeneca, as well as sits on a study advisory committee for IQVIA.

Patient consent for publication Not required. 
Ethics approval Ethical approval was given by the Regional Ethical Review Board at Karolinska Institutet (2013/1156-31/5). Informed consent is required for inclusion in the MSR.

Provenance and peer review Not commissioned; externally peer reviewed.

Data availability statement No data are available.

Open access This is an open access article distributed in accordance with the Creative Commons Attribution Non Commercial (CC BY-NC 4.0) license, which permits others to distribute, remix, adapt, build upon this work non-commercially, and license their derivative works on different terms, provided the original work is properly cited, appropriate credit is given, any changes made indicated, and the use is non-commercial. See: http://creativecommons.org/licenses/by-nc/4.0/.

ORCID iD

Kelsi A Smith http://orcid.org/0000-0002-9811-908X

\section{REFERENCES}

1 Hedström AK, Olsson T, Alfredsson L. Smoking is a major preventable risk factor for multiple sclerosis. Mult Scler 2016;22:1021-6.

2 Hedström AK, Hössjer O, Katsoulis M, et al. Organic solvents and MS susceptibility. Neurology 2018;91:e455-62.

3 Shan $\mathrm{M}$, Cheng $\mathrm{H}-\mathrm{F}$, Song $\mathrm{L}-\mathrm{z}$, et al. Lung myeloid dendritic cells coordinately induce Th1 and Th17 responses in human emphysema. Sci Transl Med 2009;1:4ra10-1.

4 Hedström AK, Bomfim IL, Barcellos LF, et al. Interaction between passive smoking and two HLA genes with regard to multiple sclerosis risk. Int J Epidemiol 2014;43:1791-8.

5 Odoardi F, Sie C, Streyl K, et al. T cells become licensed in the lung to enter the central nervous system. Nature 2012;488:675-9.

6 Bagos PG, Nikolopoulos G, loannidis A. Chlamydia pneumoniae infection and the risk of multiple sclerosis: a meta-analysis. Mult Scler 2006;12:397-411.

7 Chan JYC, Stern DA, Guerra S, et al. Pneumonia in childhood and impaired lung function in adults: a longitudinal study. Pediatrics 2015;135:607-16.

8 Sundin P-O, Udumyan R, Fall K, et al. Hospital admission with pneumonia and subsequent persistent risk of chronic kidney disease: national cohort study. Clin Epidemiol 2018;10:971-9.

9 Bergh C, Fall K, Udumyan R, et al. Severe infections and subsequent delayed cardiovascular disease. Eur J Prev Cardiol 2017;24:1958-66.

10 Ramagopalan SV, Dobson R, Meier UC, et al. Multiple sclerosis: risk factors, prodromes, and potential causal pathways. Lancet Neurol 2010;9:727-39.

11 Thacker EL, Mirzaei F, Ascherio A. Infectious mononucleosis and risk for multiple sclerosis: a meta-analysis. Ann Neurol 2006;59:499-503.

12 Montgomery S, Hiyoshi A, Burkill S, et al. Concussion in adolescence and risk of multiple sclerosis. Ann Neurol 2017;82:554-61.

13 Wijnands JMA, Zhu F, Kingwell E, et al. Five years before multiple sclerosis onset: phenotyping the prodrome. Mult Scler J 2018:1-10.

14 Montgomery S, Hillert J, Bahmanyar S. Hospital admission due to infections in multiple sclerosis patients. Eur J Neurol 2013;20:1153-60.

15 Ludvigsson JF, Andersson E, Ekbom A, et al. External review and validation of the Swedish national inpatient register. BMC Public Health 2011;11:450.

16 Hillert J, Stawiarz L. The Swedish MS registry - clinical support tool and scientific resource. Acta Neurol Scand 2015;132:11-19.

17 Bahmanyar S, Montgomery SM, Hillert J, et al. Cancer risk among patients with multiple sclerosis and their parents. Neurology 2009;72:1170-7.

18 Wijnands JMA, Kingwell E, Zhu F, et al. Infection-Related health care utilization among people with and without multiple sclerosis. Mult Scler 2017;23:1506-16.

19 Blakemore SJ, Burnett S, Dahl RE. The role of puberty in the developing adolescent brain. Hum Brain Mapp 2010;31:926-33.

20 Khundrakpam BS, Lewis JD, Zhao L, et al. Brain connectivity in normally developing children and adolescents. Neuroimage 2016;134:192-203.
21 Mills KL, Goddings A-L, Herting MM, et al. Structural brain development between childhood and adulthood: convergence across four longitudinal samples. Neuroimage 2016;141:273-81.

22 Whitaker KJ, Vértes PE, Romero-Garcia R, et al. Adolescence is associated with genomically patterned consolidation of the hubs of the human brain connectome. Proc Natl Acad Sci U S A 2016;113:9105-10

23 Bjørnevik K, Riise T, Cortese M, et al. Level of education and multiple sclerosis risk after adjustment for known risk factors: the EnvIMS study. Mult Scler 2016;22:104-11.

24 Cohen S, Janicki-Deverts D, Chen E, et al. Childhood socioeconomic status and adult health. Ann N Y Acad Sci 2010;1186:37-55.

25 Hamblin TJ, Hussain J, Akbar AN, et al. Immunological reason for chronic ill health after infectious mononucleosis. BMJ 1983;287:85-8.

26 Giovannoni G. How long is the presymptomatic phase of multiple sclerosis? Mult Scler Relat Disord 2016;7:12-13.

27 Montgomery S, Hiyoshi A, Burkill S, et al. Reply to "concussion may not cause multiple sclerosis". Ann Neurol 2017;82:652-3.

28 Endriz J, Ho PP, Steinman L. Time correlation between mononucleosis and initial symptoms of MS. Neurol Neuroimmunol Neuroinflamm 2017;4:e308-8.

29 Lin C-W, Huang Y-P, Pan S-L. Spinal cord injury is related to an increased risk of multiple sclerosis: a population-based, propensity score-matched, longitudinal follow-up study. J Neurotrauma 2015;32:655-9.

30 Wang Y, Jiang B, Guo Y, et al. Cross-Protective mucosal immunity mediated by memory Th17 cells against Streptococcus pneumoniae lung infection. Mucosal Immunol 2017;10:250-9.

31 Marqués JM, Rial A, Muñoz N, et al. Protection against Streptococcus pneumoniae serotype 1 acute infection shows a signature of Th17- and IFN- $\gamma$-mediated immunity. Immunobiology 2012;217:420-9.

32 Li W, Zhang X, Yang Y, et al. Recognition of conserved antigens by Th17 cells provides broad protection against pulmonary Haemophilus influenzae infection. Proc Natl Acad Sci U S A 2018;115:E7149-57.

33 Tzartos JS, Friese MA, Craner MJ, et al. Interleukin-17 production in central nervous system-infiltrating $T$ cells and glial cells is associated with active disease in multiple sclerosis. Am J Pathol 2008;172:146-55.

34 Tsai P-K, Hsieh M-J, Wang H-L, et al. Elevated plasma stromal-cellderived factor-1 protein levels correlate with severity in patients with community-acquired pneumonia. Dis Markers 2014;2014:1-8.

35 Rosseau S, Hocke A, Mollenkopf H, et al. Comparative transcriptional profiling of the lung reveals shared and distinct features of Streptococcus pneumoniae and influenza A virus infection. Immunology 2007;120:380-91.

36 Galli E, Hartmann FJ, Schreiner B, et al. Gm-Csf and CXCR4 define a T helper cell signature in multiple sclerosis. Nat Med 2019;25:1290-300.

37 Buljevac D, Flach HZ, Hop WCJ, et al. Prospective study on the relationship between infections and multiple sclerosis exacerbations. 2002;125:952-60.

38 Heier I, Malmström K, Sajantila A, et al. Characterisation of bronchus-associated lymphoid tissue and antigen-presenting cells in central airway mucosa of children. Thorax 2011;66:151-6.

39 Hiller AS, Tschernig T, Kleemann WJ, et al. Bronchus-associated lymphoid tissue (BALT) and larynx-associated lymphoid tissue (LALT) are found at different frequencies in children, adolescents and adults. Scand J Immunol 1998;47:159-62.

40 Tschernig T, Pabst R. Bronchus-associated lymphoid tissue (BALT) is not present in the normal adult lung but in different diseases. Pathobiology 2000;68:1-8.

41 Murley C, Friberg E, Hillert J, et al. Validation of multiple sclerosis diagnoses in the Swedish national patient register. Eur J Epidemiol 2019;34:1161-9.

42 Public Health Agency of Sweden. Vaccination statistics from primary health care centers for children (in Swedish). Available: https:// www.folkhalsomyndigheten.se/folkhalsorapportering-statistik/ statistikdatabaser-och-visualisering/vaccinationsstatistik/statistikfran-barnavardcentraler/ [Accessed 9 Jan 2019].

43 Bhuiyan MU, Snelling TL, West R, et al. Role of viral and bacterial pathogens in causing pneumonia among Western Australian children: a case-control study protocol. BMJ Open 2018;8:e020646-10. 